\section{La biogenèse du VIH-1 dévoilée}

Nolwenn Jouvenet
Aaron Diamond AIDS Research Center, Laboratory of Retrovirology, The Rockefeller University, 455 lst Avenue, 10016 New York, États-Unis.

njouvene@adarc.org
> Les récentes avancées technologiques d'imagerie cellulaire ont permis de visualiser de nombreuses étapes de la réplication des virus dans des cellules vivantes. Ainsi, en fusionnant certaines protéines virales à des protéines fluorescentes, l'attachement, la pénétration et le transport de virus dans leurs cellules hôtes ont pu être caractérisés au cours des dernières années [1]. Cependant, la biogenèse de nouveaux virus, une étape clé de la réplication virale, n'avait pas encore été visualisée jusqu'à ce jour.

\section{Assemblage plasmique} des molécules Gag de VIH-1

La biogenèse d'un virus consiste en l'adressage des composants du virus en un site cellulaire particulier, où ces composants s'assemblent pour former de nouvelles particules virales qui seront ultérieurement libérées dans le milieu extérieur [2]. Dans le cas du virus de l'immunodéficience humaine de type 1 (VIH-1), l'assemblage a lieu à la membrane plasmique des cellules hôtes [35]. La protéine virale Gag, qui est le composant principal du VIH-1, orchestre ce processus: son expression est suffisante pour engendrer la formation de nouveaux virus. La concentration et l'assemblage compact de plusieurs milliers de copies de la protéine Gag sous la membrane plasmique entraînent la déformation de celle-ci, jusqu'à l'obtention d'une vésicule d'une centaine de nanomètres de diamètre qui bourgeonne vers le milieu extérieur, puis se sépare de la cellule. Cette séparation est dépendante de l'interaction entre Gag et le complexe protéique ESCRT (endosomal sorting complex required for transport), ce dernier étant impliqué dans les séparations de membranes cellulaires, comme le bourgeonnement des vésicules internes des endosomes multivésiculaires [6], ou encore la séparation des membranes de 2 cellules filles après la division cellulaire [7]. Toutes les connaissances de la biogenèse virale sont déduites d'études biochimiques effectuées sur des populations de virus et de l'observation microscopique de virus dans des cellules fixées. Tous les aspects cinétiques de l'assemblage sont donc encore inconnus.

\section{L'onde évanescente de TIR-FM pour une imagerie de la membrane à haute résolution}

Afin d'y remédier, nous avons travaillé avec des cellules vivantes exprimant la protéine Gag fusionnée à la protéine fluorescente verte (Gag-GFP) et utilisé une technique de microscopie qui permet d'étudier avec une très grande réso- lution les évènements intervenant à la membrane plasmique [8]. Le principe de cette technique, appelée TIR-FM (Total Internal Reflection Fluorescence Microscopy) est d'illuminer l'échantillon avec une lumière dont l'angle d'incidence est tel que la lumière va être entièrement réfléchie à l'interface cellule/support en verre. Cela génère une onde évanescente faible qui pénètre dans le milieu dont I'indice de réfraction est le moins élevé (dans ce cas, la cellule) jusqu'à une distance de quelques centaines de nanomètres. En comparaison avec l'imagerie conventionnelle à champ large et même l'imagerie confocale, le contraste des images obtenues, ainsi que le rapport signal/bruit de fond sont considérablement améliorés (Figure 1). Cette technique a été développée dans les années 1980 , et elle est communément utilisée en biologie cellulaire pour étudier les évènements moléculaires qui ont lieu

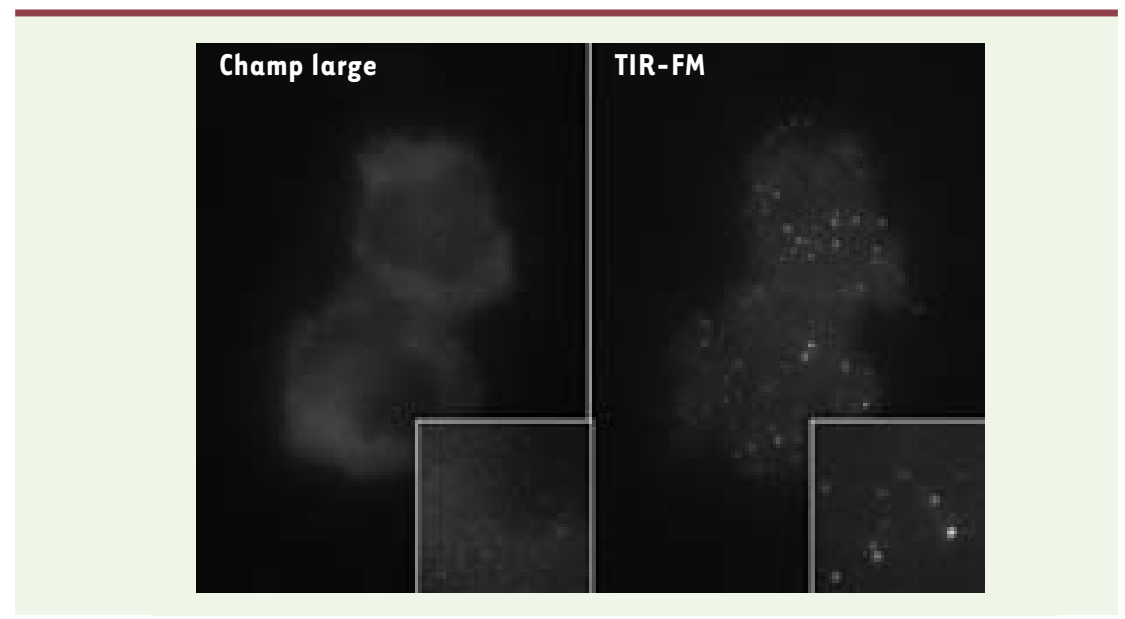

Figure 1. Visualisation des particules virales à la membrane plasmique avec la technique du TIR-FM. Une cellule vivante exprimant les protéines Gag et Gag-GFP est photographiée avec un microscope à fluorescence utilisé soit en mode conventionnel « champ large » (à gauche), soit en mode TIR-FM (à droite). La mise au point est inchangée entre les 2 prises de vues. 

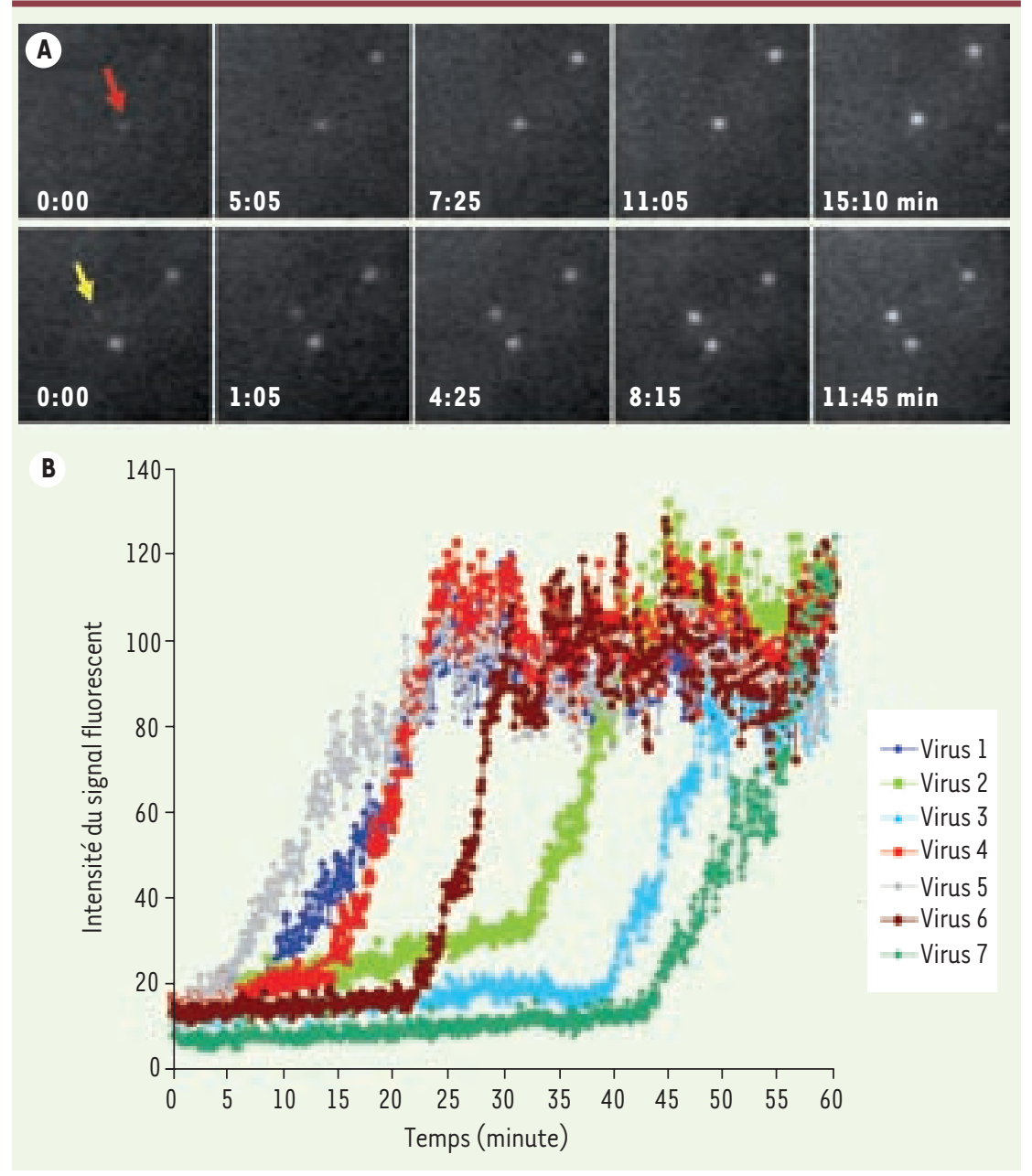

Figure 2. Assemblage du VIH-1 en temps réel. Des cellules vivantes exprimant les protéines Gag et Gag-GFP sont photographiées toutes les 5 secondes pendant 60 minutes. A. La suite d'images acquises par TIR-FM montre l'apparition de 2 virus fluorescents (flèches) à la membrane plasmique. Le temps est indiqué en minutes: secondes. $\boldsymbol{B}$. Les courbes illustrent l'évolution du signal fluorescent émis par Gag-GFP pendant l'assemblage de 7 virus à la surface d'une cellule. L'intensité du signal fluorescent est calculée par le logiciel Metamorph ; elle est donnée en unités arbitraires.

à la surface des cellules; par exemple, les mécanismes d'endocytose et d'exocytose [8].

\section{Capturer l'assemblage}

des virus en temps réel : FRET et FRAP

Nous avons ainsi détecté avec une résolution sans précédent les virus fluorescents à la membrane plasmique (Figure 1) [9]. L'une des principales difficultés était de capturer l'assemblage des virus «en direct». Nous savions, grâce à nos travaux biochimiques, que quelques heures seulement après leur plusieurs minutes, puis se stabilisait (Figure 2B). L'augmentation régulière du signal fluorescent représente très probablement la concentration et l'assemblage des protéines Gag à la surface des cellules.

Afin de confirmer cette hypothèse, nous avons utilisé la technique du FRET (Fluorescence Resonance Energy transfer). Celle-ci est basée sur les transferts d'énergie entre 2 protéines fluorescentes, par exemple la GFP et mCherry (qui émet une fluorescence rouge), et elle informe sur la proximité des protéines fusionnées à ces fluorophores [10]. En effet, plus les protéines sont rapprochées, plus les transferts d'énergie de GFP vers mCherry sont importants et plus mCherry fluoresce. Le niveau de FRET se mesure par le rapport entre le signal fluorescent émis par GFP et celui émis par mCherry. Pendant l'apparition des virus à la membrane plasmique, le niveau de FRET au sein de chaque virus augmente régulièrement pendant quelques minutes, puis il se stabilise, jusqu'à atteindre un niveau comparable à celui mesuré dans les virus libérés dans le surnageant de culture [9]. Ces données indiquent que pendant l'apparition de ces virus, les protéines Gag deviennent de plus en plus proches les unes des autres, pour atteindre un degré de concentration aussi élevé que celui détecté dans des virus complètement assemblés.

La technique du FRAP (Fluorescence Recovery after Photobleaching) a ensuite permis de mettre en évidence le recrutement actif de nouvelles protéines Gag durant ces apparitions [9]. En effet, si les particules virales sont soumises pendant leur formation à une forte application laser qui détruit irréversiblement la GFP, la fluorescence réapparaît toujours. En revanche, il n'y a pas de réapparition de fluorescence pour les virus dont la fluorescence était stable avant le photoblanchiment. Ces données indiquent que de nouvelles protéines Gag sont recrutées pendant l'apparition de chacun de ces virus et que la stabilisation du signal 
fluorescent correspond à l'arrêt du recrutement et donc, trés probablement, à l'arrêt de l'assemblage des virus.

Biogenèse du VIH-1 :

une question de minutes

Les résultats des expériences de FRET et de FRAP nous ont convaincus que les évènements que nous visualisions étaient de véritables événements d'assemblage. Nous avons alors mesuré la durée de formation de centaines de virus et nous avons ainsi déterminé que la biogenèse du VIH-l nécessite 5 à 6 minutes. Cependant, de manière surprenante, une fois l'assemblage achevé, la grande majorité des virus restaient immobiles dans le champ de vision. Afin de nous assurer que ces virus étaient correctement libérés de la membrane plasmique, nous avons fusionné Gag à la protéine Phluorin, une variante de la GFP dont l'intensité d'émission est optimale à $\mathrm{pH} 7,5$ et diminue avec l'acidification [11]. Une acidification rapide du cytoplasme des cellules peut être induite en exposant le milieu de culture à du $\mathrm{CO}_{2}$ concentré à $100 \%$ pendant 20 secondes [12]. En étudiant les conséquences de cette acidification sur la fluorescence de centaines de virus composés de milliers de copies de Gag-Phluorin, nous avons pu distinguer les virus qui étaient encore attachés à la cellule (ceux dont la fluorescence était très sensible aux changements de $\mathrm{pH}$ ) de ceux qui avaient bourgeonné (ceux dont la fluorescence était peu sensible aux changements de $\mathrm{pH}$ du cytoplasme puisque séparés de la cellule) [9]. La présence des virus dans le champ après leur bourgeonnement peut s'expliquer par leur immobilisation entre les cellules et leur support ou par leur rétention à la membrane par la protéine Tetherin [13].

Nous avons ainsi établi un système original permettant de visualiser l'assemblage de particules virales en temps réel. En visualisant simultanément Gag et d'autres composants clés du virus, comme le matériel génétique ou la protéine de l'enveloppe, ou encore Gag et le complexe protéique ESCRT, nous devrions être capable d'approfondir nos connaissances sur l'assemblage et le bourgeonnement du VIH-l. Nous espérons également utiliser ce système pour étudier la biogenèse d'autres types de virus. $\diamond$

HIV- 1 biogenesis unmasked

\section{REMERCIEMENTS}

Je remercie Paul Bieniasz et Sanford Simon pour la supervision de ces recherches, Véronique Douard pour la relecture pertinente de cette nouvelle, ainsi que l'amfAR (Foundation for AIDS Research) pour l'attribution d'une bourse postdoctorale « Mathilde Krim».

\section{RÉFÉRENCES}

1. Brandenburg B, Zhuang $X$. Virus trafficking : learning from single-virus tracking. Nat Rev Microbiol 2007 ; 5 : 197-208.

2. Corbin A, Grigorov B, Roingeard P, et al. Une nouvelle vision de l'assemblage du VIH-1. Med Sci (Paris) $2008 ; 24: 49-55$

3. Jouvenet $\mathrm{N}$, Neil SJ, Bess C, et al. Plasma membrane is the site of productive HIV-1 particle assembly. PLoS Biol 2006 ; 4 : e435.

4. Welsch S, Keppler OT, Habermann A, et al. HIV-1 buds predominantly at the plasma membrane of primary human macrophages. PLoS Pathog 2007 ; 3 : e36.

5. Finzi A, Orthwein A, Mercier J, Cohen EA. Productive human immunodeficiency virus type 1 assembly takes place at the plasma membrane. J Virol 2007; 81 : 7476-90.

6. Von Schwedler UK, Stuchell M, Müller B, et al. The protein network of HIV budding. Cell 2003; 114 : 70113.

7. Carlton JG, Martin-Serrano J. Parallels between cytokinesis and retroviral budding : a role for the ESCRT machinery. Science 2007 ; 316 : 1908-12.

8. Jaiswal JK, Simon SM. Imaging single events at the cell membrane. Nat Chem Biol 2007 ; 3 : 92-8.

9. Jouvenet N, Bieniasz PD, Simon SM. Imaging the biogenesis of individual HIV-1 virions in live cells. Nature 2008 ; 454 : 236-40.

10. Trugnan $G$, Fontanges $P$, Delautier D, Ait-Slimane T. FRAP, FLIP, FRET, BRET, FLIM, PRIM... De nouvelles techniques pour voir la vie en couleur ! Med Sci (Paris) $2004 ; 20: 1027-34$.

11. Miesenbock, G, De Angelis DA, Rothman JE. Visualizing secretion and synaptic transmission with $\mathrm{pH}$-sensitive green fluorescent proteins. Nature 1998 ; 394 : 192-5.

12. Simon S, Roy D, Schindler M. Intracellular pH and the control of multidrug resistance. Proc Natl Acad Sci USA 1994 ; 91 : 1128-32.

13. Neil SJ, Zang T, Bieniasz PD. Tetherin inhibits retrovirus release and is antagonized by HIV-1 Vpu. Nature $2008 ; 451: 425-30$.

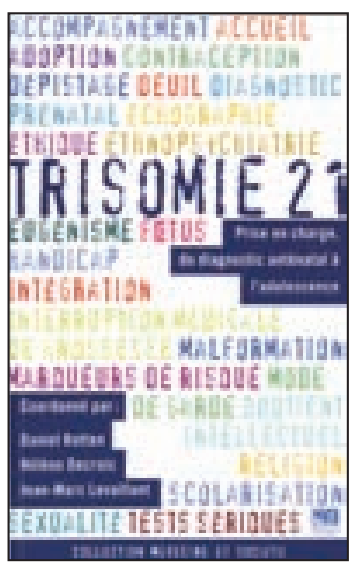

ISBN : 2-84254-105-7 248 pages

\section{Bon de commande}

À retourner à EDK, 2, rue Troyon - 92316 Sèvres Cedex

Tél. : 0155641393 - Fax : 0155641394 - E-mail : edk@edk.fr

NOM :

Prénom :

Adresse :

Code postal :

Ville :

Pays :

Fonction :

Je souhaite recevoir l'ouvrage Trisomie $21: 15 €+3 €$ de port $=\mathbf{1 8} €$ TTC

en ................. exemplaire, soit un total de ....................................... €

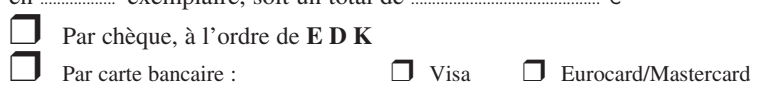

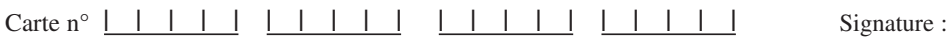

Date d'expiration: $\quad \leq 1|1|$

$\mathrm{N}^{\circ}$ de contrôle au dos de la carte : 